\title{
KRULL-SCHMIDT FAILS FOR ARTINIAN MODULES
}

\author{
ALBERTO FACCHINI, DOLORS HERBERA, LAWRENCE S. LEVY, AND PETER VÁMOS
}

(Communicated by Ken Goodearl)

\begin{abstract}
We prove that the Krull-Schmidt theorem fails for artinian modules. This answers a question asked by Krull in 1932. In fact we show that if $S$ is a module-finite algebra over a semilocal noetherian commutative ring, then every nonunique decomposition of every noetherian $S$-module leads to an analogous nonunique decomposition of an artinian module over a related non-noetherian ring. The key to this is that any such $S$ is the endomorphism ring of some artinian module.
\end{abstract}

Krull asked, in [K '32, p. 38], whether the Krull-Schmidt theorem holds for artinian modules; that is, whether $M_{1} \oplus \ldots \oplus M_{m} \cong N_{1} \oplus \ldots \oplus N_{n}$ with each $M_{i}$ and $N_{i}$ an indecomposable artinian right module over some ring $R$, implies that $m=n$ and, after a suitable renumbering of the summands, each $M_{i} \cong N_{i}$.

Warfield showed [W '69, Proposition 5] that the answer is "yes" when the ring $R$ is either right noetherian or commutative. He did this by showing that, over any ring, any artinian indecomposable module that is a union of modules of finite length has a local endomorphism ring. [Recall that direct sums of indecomposable modules with local endomorphism rings have unique directsum decompositions, even when the direct sum contains infinitely many terms.]

Subsequently, Camps and Dicks showed that the endomorphism ring of any artinian module is semilocal (i.e. semisimple artinian modulo its Jacobson radical) [CD '93]. They concluded that artinian modules cancel from direct sums; that is, if $M \oplus A \cong M \oplus B$ with $M$ artinian, then $A \cong B$.

In this note we answer Krull's question by showing that the Krull-Schmidt theorem fails for general artinian modules. The idea of the proof is that decompositions of modules correspond to decompositions of their endomorphism ring, in a natural way. This reduces the question to that of what kinds of

Received by the editors October 25, 1993 and, in revised form, May 2, 1994.

1991 Mathematics Subject Classification. Primary 16P20, 16D70.

Key words and phrases. Krull-Schmidt, artinian module, direct sum decomposition, endomorphism ring.

The second author's research was supported by a postdoctoral fellowship from the Ministerio de Educación y Ciencia of Spain and the Fulbright Foreign Scholarship Board and by a grant of the DGICYT (Spain). It was done in the Mathematics Department of Rutgers University, and she wishes to thank the University and its faculty for their hospitality.

This research was begun at a meeting on module theory at the Mathematisches Forschungsinstitut Oberwolfach, Germany. 
semilocal rings can occur as endomorphism rings of artinian modules. We apply a result of Camps and Menal to show that all module-finite algebras over semilocal noetherian rings can occur in this way. A special case of our main result is that all decompositions of noetherian modules over the semilocal rings that occur in integral representation theory yield corresponding decompositions of artinian modules over nonnoetherian rings (see Theorem 1.5); and we quote known examples to show that such decompositions can be far from unique.

In $\S 2$ we supplement Camps and Dicks' cancellation result by showing that for artinian modules $X, Y$ we have (i) $X^{n} \cong Y^{n} \Rightarrow X \cong Y$; and (ii) $X$ has only finitely many isomorphism classes of direct summands. These are both easy consequences of the fact that the endomorphism ring is semilocal.

\section{NONUNIQUENESS}

The notation $\operatorname{End}(M)$ denotes the endomorphism ring of a right module $M$ over some ring, usually specified by the context. We write endomorphisms on the left. Recall that a left module $M$ over a ring $R$ cogenerates a left $R$-module $X$ if $X$ is contained in a direct product of copies of $M$. Equivalently, for every nonzero $x \in X$, there is an $R$-homomorphism $\phi: X \rightarrow M$ such that $\phi(x) \neq 0$. $M$ is called a (left $R$ )-cogenerator if $M$ cogenerates every left $R$-module. It is easy to prove the following well-known fact: If $M$ is an injective left $R$-module that contains an isomorphic copy of every simple left $R$-module, then $M$ is an $R$-cogenerator.

Our starting point is the following criterion of Camps and Menal for the existence of artinian modules with specified endomorphism rings.

Proposition 1.1 [CF, Proposition 1.3]. Let $E$ be a subring of a right artinian ring $A$. Suppose that there exists a ring $R$ and an $E$-R-bimodule $N$ such that $N_{R}$ is artinian and ${ }_{E} N$ cogenerates ${ }_{E}(A / E)$. Then $E \cong \operatorname{End}(M)$ for some artinian cyclic right module $M$ over some ring.

We include a proof of the following known lemma, since it is cleaner than an awkward chain of references to its various parts.

Lemma 1.2. Every module-finite algebra over a commutative noetherian ring can be imbedded in an artinian ring.

Proof. Let $R$ be the commutative noetherian ring. If the lemma is false, then by noetherian induction we can find a module-finite $R$-algebra $E$ which cannot be embedded in an artinian ring, but such that every proper homomorpic image of $E$ can be so embedded. To complete the proof it suffices to find nonzero ideals $I$ and $E t$ of $E$ such that $I \cap E t=0$, for then $E$ imbeds in $E / I \times E / E t$; and since each factor imbeds in an artinian ring, so does $E$, a contradiction.

We may assume that $R$ is contained in the center of $E$. Let $S$ be the complement of the union of the finite number of minimal primes of $R$, and set

$$
I=\operatorname{Ker}\left(E \rightarrow S^{-1} E\right)=\{e \in E \mid s e=0 \text { for some } s \in S\} .
$$

$S^{-1} R$ is an artinian ring, and therefore so is its module-finite algebra $S^{-1} E$. Since $E$ does not imbed in an artinian ring, we have $I \neq 0$. Since $E$ is noetherian, $I$ is a finitely generated ideal so there is a $t \in S$ such that $t I=$ 
$I t=0$. The ring $R / R t$ is artinian, and therefore so is its module-finite algebra $E / E t$. Therefore $E t \neq 0$.

Suppose $i=e t \in I \cap E t$. Then $0=i t=e t^{2}$ and therefore $e \in I$, which implies that $0=e t=i$. Therefore $I \cap E t=0$.

Corollary 1.3. Let $E$ be a module-finite algebra over a semilocal noetherian commutative ring. Then $E \cong \operatorname{End}(M)$ for some artinian cyclic right module $M$ over some ring.

Proof. First we note that $E \subseteq A$ for some (right and left) artinian ring $A$, by Lemma 1.2.

Now let $R$ be the semilocal noetherian ring over which $E$ is a modulefinite algebra. Let $I_{1}, \ldots, I_{n}$ be the maximal ideals of $R$, and for each $j$ let $E\left(R / I_{j}\right)$ denote the injective envelope of the $R$-module $R / I_{j}$. Then the $R$-module $C=\bigoplus_{j} E\left(R / I_{j}\right)$ is an injective $R$-cogenerator and is an artinian $R$-module [SV' 72 , Theorem 4.30].

We claim, as is remarked in [V'77, §2], that the left $E$-module $N=$ $\operatorname{Hom}_{R}(E, C)$ is an $E$-cogenerator. To see this, suppose that $0 \neq k_{0} \in K$ for some left $E$-module $K$. We need to find an $E$-homomorphism $f: K \rightarrow N$ such that $f\left(k_{0}\right) \neq 0$. Since $C$ is an $R$-cogenerator, there is an $R$-homomorphism $\phi: K \rightarrow C$ such that $\phi\left(k_{0}\right) \neq 0$. Define an $E$-homomorphism $f: K \rightarrow N$ by $f(k)(e)=\phi(e k)$. Then $f\left(k_{0}\right) \neq 0$, completing the proof of the claim.

Now we apply Proposition 1.1. Since $E$ is a subring of the artinian ring $A$ and ${ }_{E} N$ is an $E$-cogenerator, it suffices to prove that $N_{R}$ is artinian.

Since ${ }_{R} E$ is finitely generated, there is an exact sequence of left $R$-modules $R^{n} \rightarrow E \rightarrow 0$. Applying the functor $\operatorname{Hom}_{R}(-, C)$ yields an exact sequence of right $R$-modules

$$
0 \rightarrow N=\operatorname{Hom}_{R}\left({ }_{R} E,{ }_{R} C_{R}\right) \rightarrow \operatorname{Hom}_{R}\left(R^{n},{ }_{R} C_{R}\right) \cong C_{R}^{n} .
$$

Since $C_{R}$ is artinian, $N_{R}=\operatorname{Hom}_{R}\left({ }_{R} E,{ }_{R} C_{R}\right)$ is artinian too, and this completes the proof of our corollary.

The precise link between endomorphism rings and decompositions of modules is given by the following special case of a well-known lemma.

Lemma 1.4. Let $M$ be a right module over some ring $S$ and $E=\operatorname{End}(M)$. (Recall that we write endomorphisms on the left.) Then

(i) $A$ bijection between the set of all finite direct-sum decompositions $M_{S}=$ $\bigoplus_{i} M_{i}$ and the set of all finite direct-sum decompositions $E_{E}=\bigoplus_{i} E_{i}$ is given by $E_{i}=e_{i} E$ where $e_{i}$ is the projection map $M \rightarrow M_{i}$ viewed as an element of $E$. Moreover, $M_{i}$ is an indecomposable $S$-module $\Longleftrightarrow E_{i}$ is an indecomposable E-module.

(ii) $M_{i} \cong M_{j}$ as $S$-modules $\Longleftrightarrow E_{i} \cong E_{j}$ as $E$-modules.

Proof. This easily proved lemma results from the fact that any finite decomposition $M=\bigoplus_{i} M_{i}$ yields the decomposition $E=\bigoplus_{i} \operatorname{Hom}_{S}\left(M, M_{i}\right)$, while any decomposition $E_{E}=\bigoplus_{i} E_{i}$ yields the decomposition $M=\bigoplus_{i} E_{i} M=$ $\bigoplus_{i}\left(E_{i} \otimes_{E} M\right)$.

The following is our main result, stating that decompositions of certain noetherian modules yield decompositions of artinian modules. 
Theorem 1.5. Let $S$ be a module-finite algebra over a semilocal noetherian commutative ring $R$, and consider a direct-sum relation

$$
\bigoplus_{i=1}^{m} M_{i}=M=\bigoplus_{i=m+1}^{n} M_{i} \quad\left(\text { each } M_{i} \text { indecomposable }\right)
$$

of noetherian right $S$-modules. Then there is a direct-sum relation of this same form:

$$
\bigoplus_{i=1}^{m} M_{i}^{\prime}=M^{\prime}=\bigoplus_{i=m+1}^{n} M_{i}^{\prime} \quad\left(\text { each } M_{i}^{\prime} \text { indecomposable }\right)
$$

involving artinian cyclic right modules $M_{i}^{\prime}$ (over some ring) such that $M_{i}^{\prime} \cong M_{j}^{\prime}$ $\Longleftrightarrow M_{i} \cong M_{j}$.

Proof. Let $E=\operatorname{End}_{S}(M)$. Since $S$ is a module-finite $R$-algebra and $R$ is noetherian, $E$ is again a module-finite $R$-algebra. Lemma 1.4 yields a pair of direct-sum decompositions of the right $E$-module $E$, of the same form as (1.5.1):

$$
\bigoplus_{i=1}^{m} E_{i}=E=\bigoplus_{i=m+1}^{n} E_{i} \quad\left(\text { each } E_{i} \text { indecomposable }\right)
$$

By Corollary 1.3 , the module-finite $R$-algebra $E$ is isomorphic to $\operatorname{End}\left(M^{\prime}\right)$ for some artinian cyclic module $M^{\prime}$ over some ring. An application of Lemma 1.4 to decompositions (1.5.3) now yields the desired decompositions (1.5.2) of $M^{\prime}$.

We close this section with two examples, showing quite different types of failure of Krull-Schmidt for artinian modules.

Example 1.6 (nonuniqueness of the number of indecomposable summands). For every positive integer $n$ there is an artinian module $M$ that is the direct sum of 2 indecomposable modules, and also the direct sum of three indecomposable modules, and ..., and also the direct sum of $n$ indecomposable modules.

Proof. By Theorem 1.5 it suffices to find a noetherian right module $M$, with the desired decompositions, over a ring $S$ that is a module-finite algebra over a semilocal commutative noetherian ring $R$.

This is done in [L '83, Example 3.2]. In fact, this can be done with $R$ the localization of the ring of integers at the complement of any four maximal ideals, and $S$ an $R$-subalgebra of a direct product of copies of $R$. [In Notation 0.2 of that paper, let $Z$ denote the localization of the ring of integers at the complement of four distinct prime numbers $p, q, r, s$. Then, in diagram (G), below Notation 0.2 , choose each $p_{i} \in\{p, q, r, s\}$ such that the two or four primes that meet at each vertex of $(\mathrm{G})$ are distinct. Finally, let $S$ be the ring called $R$ in (1.1) of that paper.]

Example 1.7 (simple failure of Krull-Schmidt). There exist indecomposable, pairwise nonisomorphic artinian modules $M_{i}$ such that $M_{1} \oplus M_{2} \cong M_{3} \oplus M_{4}$. 
Proof. A suitable noetherian example is provided in [L '83, Example 5.2] (and many other places), and can be converted to an artinian example as in Example 1.6 .

\section{OTHER DIRECT-SUM PROPERTIES}

As already mentioned, Camps and Dicks proved that artinian modules cancel from direct sums. In more detail: They showed [CD'93, Corollary 6] that artinian modules have semilocal endomorphism rings; a well-known theorem of $H$. Bass states that semilocal rings have 1 in their stable range; and a theorem of E. G. Evans [E'73] states that 1 in the stable range of $\operatorname{End}(M)$ (for $M$ a module over any ring) is a sufficient condition for $M$ to cancel from direct sums.

In this section we obtain two additional direct-sum properties of modules with semilocal endomorphism rings.

Proposition 2.1. The following hold for all modules $X, Y$ with semilocal endomorphism rings, and all positive integers $n$.

(i) ( $n^{\text {th }}$ root uniqueness) $X^{n} \cong Y^{n} \Rightarrow X \cong Y$.

(ii) $X$ has only finitely many isomorphism classes of direct summands. If $X$ is artinian this number is $\leq 2^{\lambda}$ where $\lambda$ is the composition length of the socle of $X$.

Proof. (i) Rewrite the first isomorphism in (i) as a pair of internal decompositions $M=\bigoplus_{i=1}^{n} X_{i}=\bigoplus_{i=1}^{n} Y_{i}$ where each $X_{i} \cong X$ and $Y_{i} \cong Y$, and use Lemma 1.4 to replace these decompositions by decompositions of the semilocal endomorphism ring $E=\operatorname{End}(M)$. This yields two decompositions $E=$ $\bigoplus_{i=1}^{n} d_{i} E=\bigoplus_{i=1}^{n} e_{i} E$ where the $e_{i}$ are orthogonal idempotents whose sum is 1 and every $e_{i} E \cong e_{j} E$; and analogous statements apply to the $d_{i}$. It now suffices to prove that $d_{1} E \cong e_{1} E$.

Let $\bar{E}=E / J$, where $J$ denotes the Jacobson radical of $E$, and recall that for idempotents $d, e \in E$ we have $d E \cong e E \Longleftrightarrow \bar{d} \bar{E} \cong \bar{e} \bar{E}$ [AF '73, 17.18]. Then the two decompositions of $E$ in the previous paragraph yield a pair of decompositions $\bar{E}=\bigoplus_{i=1}^{n} \bar{d}_{i} \bar{E}_{i}=\bigoplus_{i=1}^{n} \bar{e}_{i} \bar{E}_{i}$ in which every $\bar{d}_{i} \bar{E} \cong \bar{d}_{j} \bar{E}$ and $\bar{e}_{i} \bar{E} \cong \bar{e}_{j} \bar{E}$. But since $E$ is semilocal, the ring $\bar{E}$ is semisimple artinian, and therefore $\bar{d}_{1} \bar{E} \cong \bar{e}_{1} \bar{E}$. Therefore $d_{1} E \cong e_{1} E$ as desired.

(ii) As in the proof of part (i), the number of nonisomorphic direct summands of $X$ is the same as the number of nonisomorphic direct summands of $E=$ End $(X)$. Every direct summand of $E_{E}$ has the form $e E$ for some $e=e^{2} \in E$. Hence it suffices to check that $E$ has only a finite number of nonisomorphic idempotent-generated right ideals $e E$ and-if $X$ is artinian-this number is $\leq 2^{\lambda}$.

Let $\bar{E}=E / J$ as before. Then it suffices to show that $\bar{E}$ has at most finitely many nonisomorphic idempotent-generated right ideals $\bar{e} \bar{E}$ and-if $X$ is artinian-this number is $\leq 2^{\lambda}$. Since the ring $\bar{E}$ is semisimple artinian, the number of nonisomorphic such right ideals of $\bar{E}$ is $\leq 2^{\mu}$ where $\mu$ is the cornposition length of $\bar{E}_{\bar{E}}$.

Thus it now suffices to show that, if $X$ is artinian, $\mu \leq \lambda$, and this is done in [CD '93, Corollary 6]. 
Remarks 2.2. (i) Herbera and Shamsuddin [HS] have recently shown that the endomorphism ring of any linearly compact module is semilocal, generalizing Camps and Dicks' result about artinian modules. Therefore linearly compact modules have the $n^{\text {th }}$ root uniqueness property, have only finitely many isomorphism classes of direct summands, and cancel from direct sums.

(ii) Goodearl and Warfield [GW'76] have some results with a flavor similar to ours. They obtain cancellation and $n^{\text {th }}$ root results for suitable modules over suitable rings that are von Neumann regular modulo their Jacobson radical. This is generalized by Estes and Guralnick in [EG '82].

\section{REFERENCES}

[AF '73] F. W. Anderson and K. Fuller, Rings and Categories of Modules, Springer, New York, 1973.

[CD '93] R. Camps and W. Dicks, On semilocal rings, Israel J. Math. 81 (1993), 203-211.

[CF] R. Camps and A. Facchini, The Prüfer rings that are endomorphism rings of Artinian modules, Comm. Algebra 22 (1994), 3133-3157.

[EG '82] D. R. Estes and R. M. Guralnick, Module equivalences: local to global when primitive polynomials represent units, J. Algebra 77 (1982), 138-157.

[E '73] E. G. Evans, Jr., Krull-Schmidt and cancellation over local rings, Pacific J. Math. 46 (1973), 115-121.

[GW '76] K. R. Goodearl and R. B. Warfield, Jr., Algebras over zero-dimensional rings, Math. Ann. 223 (1976), 157-168.

[HS] D. Herbera and A. Shamsuddin, Modules with semilocal endomorphism rings, Proc. Amer. Math. Soc. (to appear).

[K '32] W. Krull, Matrizen, Moduln und verallgemeinerte Abelsche Gruppen im Bereich der ganzen algebraischen Zahlen, Heidelberger Akad. Wiss. 2 (1932), 13-38.

[L '83] L. S. Levy, Krull-Schmidt uniqueness fails dramatically over subrings of $Z \oplus Z \oplus \cdots \oplus Z$, Rocky Mountain J. Math. 13 (1983), 659-678.

[SV '72] D. W. Sharpe and P. Vámos, Injective modules, Cambridge Univ. Press, Cambridge, 1972.

[V '77] P. Vámos, Rings with duality, Proc. London Math. Soc. (3) 35 (1977), 275-289.

[W '69] R. B. Warfield, Jr., A Krull-Schmidt theorem for infinite sums of modules, Proc. Amer. Math. Soc. 22 (1969), 460-465.

Dipartimento di Matematica e Informatica, Università di Udine, 33100 Udine, Italy

E-mail address: facchini@dimi.uniud.it

Departament de Matemàtiques, Universitat Autònoma de Barcelona, 08193 BellaTERRA (BARCELONa), SPAIN

E-mail address: dolors@mat.uab.es

Department of Mathematics, University of Wisconsin, Madison, Wisconsin 53706-1388 E-mail address: levy@math.wisc.edu

Pure Mathematics Department, Exeter University, Exeter EX4 4QE, England

E-mail address: vamos@maths.exeter.ac.uk 